\title{
Effect of Folic Acid therapy on Homocysteine Level in patients with Atherosclerosis or Buerger's Disease and in Healthy individuals: A clinical trial
}

\author{
Mohammad Hadi Saeed Modaghegh ${ }^{1}$, Hassan Ravari² ${ }^{2}$ Mohammad Zare Haghighi ${ }^{3}$, Ata'ollah Rajabnejad ${ }^{4}$
}

${ }^{1}$ M.D., Vascular Surgeon, Professor, Department of Vascular Surgery, Vascular and Endovascular Surgery Research Center, School of Medicine, Mashhad University of Medical Sciences, Mashhad, Iran

${ }^{2}$ M.D., Vascular Surgeon, Associate Professor, Department of Vascular Surgery, Vascular and Endovascular Surgery Research Center, School of Medicine, Mashhad University of Medical Sciences, Mashhad, Iran

${ }^{3}$ M.D., Plastic Surgeon, Department of Vascular Surgery, Vascular and Endovascular Surgery Research Center, School of Medicine, Mashhad University of Medical Sciences, Mashhad, Iran

${ }^{4}$ M.D., Department of Vascular Surgery, Vascular and Endovascular Surgery Research Center, School of Medicine, Mashhad University of Medical Sciences, Mashhad, Iran

\section{Type of article: Original}

\begin{abstract}
Background: Hyperhomocysteinemia is considered a risk factor for atherosclerosis and some other vascular diseases such as Buerger's disease.

Objective: The aim of this study was to measure the Homocysteine levels in 3 different groups of participants (Buerger's disease, atherosclerosis patients, and healthy cases) and determine the therapeutic effect of folic acid therapy on homocysteine levels for these three groups.

Methods: This nonrandomized clinical trial study was conducted in the vascular and endovascular surgery research center of Mashhad University of Medical Sciences in Mashhad, Iran. This interventional study consisted of 44 participants of which 22 patients had Buerger's disease and a control group of 22 healthy individuals, all of which were enrolled in this study. All of the study's participants had their serum homocysteine levels measured both before and after 12 weeks of folic acid ( $5 \mathrm{mg} /$ day) therapy. The data analysis used fo data analysis was a Chi square and t-test or their non-parametrical equivalents for data analysis by means of Statistical Package for the Social Sciences (SPSS) version 16.

Results: The homocysteine levels were found to be significantly higher in patients with Buerger's disease as compared to other groups before treatment with folic acid (Buerger $=21.8 \pm 8.5 \mathrm{Mm} / \mathrm{L}$, atherosclerosis $=17.3 \pm$ 6.9 , healthy $=13.8 \pm 3.1 ; \mathrm{p}<0.001)$. After treatment with folic acid at $5 \mathrm{mg} /$ daily for 12 weeks, the new plasma homocysteine levels did not show any significant difference $(\mathrm{p}=0.38)$ between the Buerger's disease group $(14.6$ $\pm 4.5 \mathrm{Mm} / \mathrm{L})$ and atherosclerosis group $(13.9 \pm 4.7)$, but it was found to besignificantly higher in both groups when compared to the healthy group $(10.7 \pm 3.9, \mathrm{p}<0.05)$. The plasma homocysteine level was reduced significantly when compared to its initial level in all 3 groups. The comparison of differences among three groups was found not to be significant $(\mathrm{p}=0.41)$.

Conclusions: It seems that supplementary therapy with folic acid at a dose of $5 \mathrm{mg}$ daily may reduce the serum homocysteine levels significantly and may have a role in the development of vascular diseases such as Buerger's disease. We suggest that folic acid should be considered as a routine agent in the Buerger's disease therapeutic
\end{abstract} regime.

Clinical trial registration: The trial was registered at the Thai Clinical Trials Registry (http://www.clinicaltrials.in.th) with the ID: TCTR20160601003.

Funding: This study was not funded by any organization.

Keywords: Buerger's disease; Atherosclerosis; Homocysteine; Folic acid

\section{Corresponding author:}

Associate Professor Dr. Hassan Ravari, Department of Vascular Surgery, Mashhad University of Medical Sciences, Mashhad, Iran. Tel: +98.5138525311, Fax: +98.5138525311, Email: Ravarih@mums.ac.ir

Received: March 28, 2016, Accepted: June 06, 2016, Published: October 2016

iThenticate screening: June 06, 2016, English editing: July 25, 2016, Quality control: September 01, 2016

(C) 2016 The Authors. This is an open access article under the terms of the Creative Commons Attribution-NonCommercialNoDerivs License, which permits use and distribution in any medium, provided the original work is properly cited, the use is non-commercial and no modifications or adaptations are made. 


\section{Introduction}

Homocysteine (Hcy) is an amino acid that was discovered in 1932 by Vigneaud that is formed as a product of the transmethylation of methionine, an essential amino acid. Hyperhomocysteinemia (in values greater than 15 micromoles per liter) is typically found in more than $60 \%$ of patients who have a form of vascular disease while its prevalence in the general population is estimated to be about 1\%. Hyperhomocysteinemia can have an effect on endothelial function and integrity, so it is a risk factor for atherosclerosis, morbidity, and mortality commonly associated with cardiovascular diseases $(1,2)$. Hyperhomocysteinemia is a result of either impaired enzymatic function or a deficiency of essential vitamins (folic acid, B6, B12) or both; hence, it can be treated with vitamin supplements (3). Limb ischemia, which is considered as a major complication in a variety of vascular diseases, can occur during atherosclerosis or thrombophilic disorders such as Buerger's disease (4). Buerger's disease, also known as thromboangiitis obliterans, is an idiopathic inflammatory process involving medium-sized arteries and veins that may lead to blockage and reduced blood flow, occurring mostly in the hands and feet (5). Serum homocysteine level tends to be higher in peripheral atherosclerosis, Buerger's disease, and deep venous thrombosis as compared to healthy persons $(4,6)$. There is a universal consensus on the positive relation between plasma homocysteine levels and the risk of vascular diseases of both the coronary and peripheral types. Several factors found to affect the plasma homocysteine levels include: folic acid, vitamin B12, and vitamin B6 status (7, 8), male sex, age, smoking, blood pressure, serum levels of cholesterol, and creatinine $(9,10)$. Although it has not been proved yet, reducing the total plasma homocysteine levels through folic acid and/or vitamin B12 consumption could lead to a decrease in the risk of vascular diseases in hyperhomocysteinemic patients (11). This study aimed to evaluate the effect of such a folic acid therapy on the homocysteine level of patients with atherosclerosis and Buerger`s disease.

\section{Material and Methods}

\subsection{Study design}

This nonrandomized clinical trial study was conducted in the vascular and endovascular surgery research center of Mashhad University of Medical Sciences in Mashhad, Iran between October 2010 and February 2011.

\subsection{Participants}

Sixty-six participants were enrolled in this study forming three groups, 22 patients suffering with lower limb ischemia due to atherosclerosis, 22 patients suffering with Buerger's disease, as well as 22 healthy persons..

\subsection{Inclusion and Exclusion criteria}

Patients who fitted all 5 shionoya criteria with their diagnosis also considered definite by an expert group of vascular surgeons were enrolled in the Buerger's disease group. Also, in the atherosclerosis group, the diagnosis was made through the presentation of ischemic symptoms followed by angiography. The exclusion criteria were havng an age under 18 years, alcohol consumption, recent use of methotrexate, trimethoprim, phenytoin, carbamazepine, or theophylline, and underlying diseases such as malignancy, systemic diseases, systemic lupus erythematosus, rheumatoid arthritis, and hypothyroidism. To minimize the possible interactions of confounding factors, vitamin B12, $1000 \mathrm{U} / \mathrm{w}$, was intravenously administered to all of the participants for one week prior to the beginning of the study.

\subsection{Procedures and outcomes}

A questioner form, including demographic variables, medical history, drug history, recent or past history of smoking, and socioeconomic status was filled out. The homocysteine plasma level was measured in all 66 patients at a time between 8 to 11 o'clock in the morning, before which the patients were asked to refrain from smoking, and to perform 30 minutes of hard exercising. Blood samples were stored in a cold box directly following the venipuncture and were protected from direct light exposure. They were centrifuged at 4 degrees Celsius for 15 minutes and then stored at -4 degrees Celsius. A homocysteine assay was performed using Axis ${ }^{\circledR}$ Homocysteine Enzyme Immunoassay (EIA) kit (UK). Then, all three groups received a daily dose of $5 \mathrm{mg}$ oral folic acid for 12 weeks. At the end of the 12 th week, the serum homocysteine level was measured again under the same conditions.

\subsection{Ethics}

This study is approved by the regional ethics committee of Mashhad University of Medical Sciences and all of the patients have signed an informed consent (Research project number: 2100). 


\subsection{Statistical analysis}

Statistical Package for the Social Sciences (SPSS) version 16 was used to analyze the data. For the quantitative variables, the statistical procedures that were selected for this analysis were the Mann Whitney or Kruskal-Wallis as the data did not follow a normal distribution. The categorical variables were analyzed using a Chi square test. A P value was less than 0.05 and, therefore, was considered significant.

\section{Results}

In this clinical trial, 83 participants were enrolled, 17 of whom either refused further cooperation or were excluded from the study. In each of three groups, there were 22 participants who received folic acid therapy. No one quit during the study and all were analyzed at the end. Figure 1 shows the CONSORT flow diagram for the study. The mean age of the participants was $48.6 \pm 17.9$ years in Buerger's disease group $(n=22), 63.5 \pm 28.2$ years in the atherosclerosis group $(\mathrm{n}=22)$ and $57.2 \pm 18.7$ years in healthy group $(\mathrm{n}=22)$. The Buerger's disease patients were significantly younger compared to the atherosclerotic or the healthy cases $(\mathrm{p}=0.018)$. Most of the participants were males, 21(95.5\%) in Buerger's disease group, $19(86.4 \%)$ in the atherosclerosis group, and $15(68.2 \%)$ in the healthy group $(p=0.06)$. Active smokers made up $90.9 \%$ of the Buerger's disease patients while their smoking rates were $31.8 \%$ and $22.7 \%$ in atherosclerosis and healthy groups, respectively $(p<0.001)$. Also, fifteen of the Buerger's patients $(68.2 \%)$ were addicted to opium. The underlying co-morbidities were significantly higher in the atherosclerotic patients $(\mathrm{p}=0.002)$ (Table 1$)$. The initial measured homocysteine plasma level was significantly higher in the Buerger's disease group as compared to the atherosclerotic patients and the healthy cases $(\mathrm{p}<0.001)$. At the end of 12 weeks of treatment with a folic acid supplement ( $5 \mathrm{mg}$ daily), the new plasma homocysteine level did not show a significant difference $(\mathrm{p}=0.38)$ between the Buerger's disease and atherosclerosis groups, but both were significantly higher compared to the healthy group $(\mathrm{p}<0.05)$. The results are displayed in Table 2 . Considering the fact that smoking has a great effect on the plasma levels of homocysteine, its levels were compared between the cigarette smokers' population with Buerger's disease (90.9\%), atherosclerosis (31.8\%), and the healthy (22.7\%) groups. The primary plasma level of homocysteine did not show a statistically significant difference among the cigarette smoker population of the 3 groups $(\mathrm{p}=0.075)$. With regard to the effects of age itself on the level of plasma homocysteine, the initial homocysteine level of plasma was compared in Buerger's disease patients and atherosclerosis patients who were younger than 50 years of age. The difference in their plasma level was found not to be statistically significant $(\mathrm{p}=0.318)$. A Wilcoxon test revealed a statistically significant decrease in homocysteine levels in all three groups after treatment with folic acid (Buerger's disease $(p=0.003)$, athrosclerosis $(\mathrm{p}<0.001)$ and healthy $(\mathrm{p}<0.001)$. This means that treatment with folic acid has resulted in a remarkable decrease in homocysteine levels. A comparison of the differences among the three groups was not significant $(p=0.41)$.

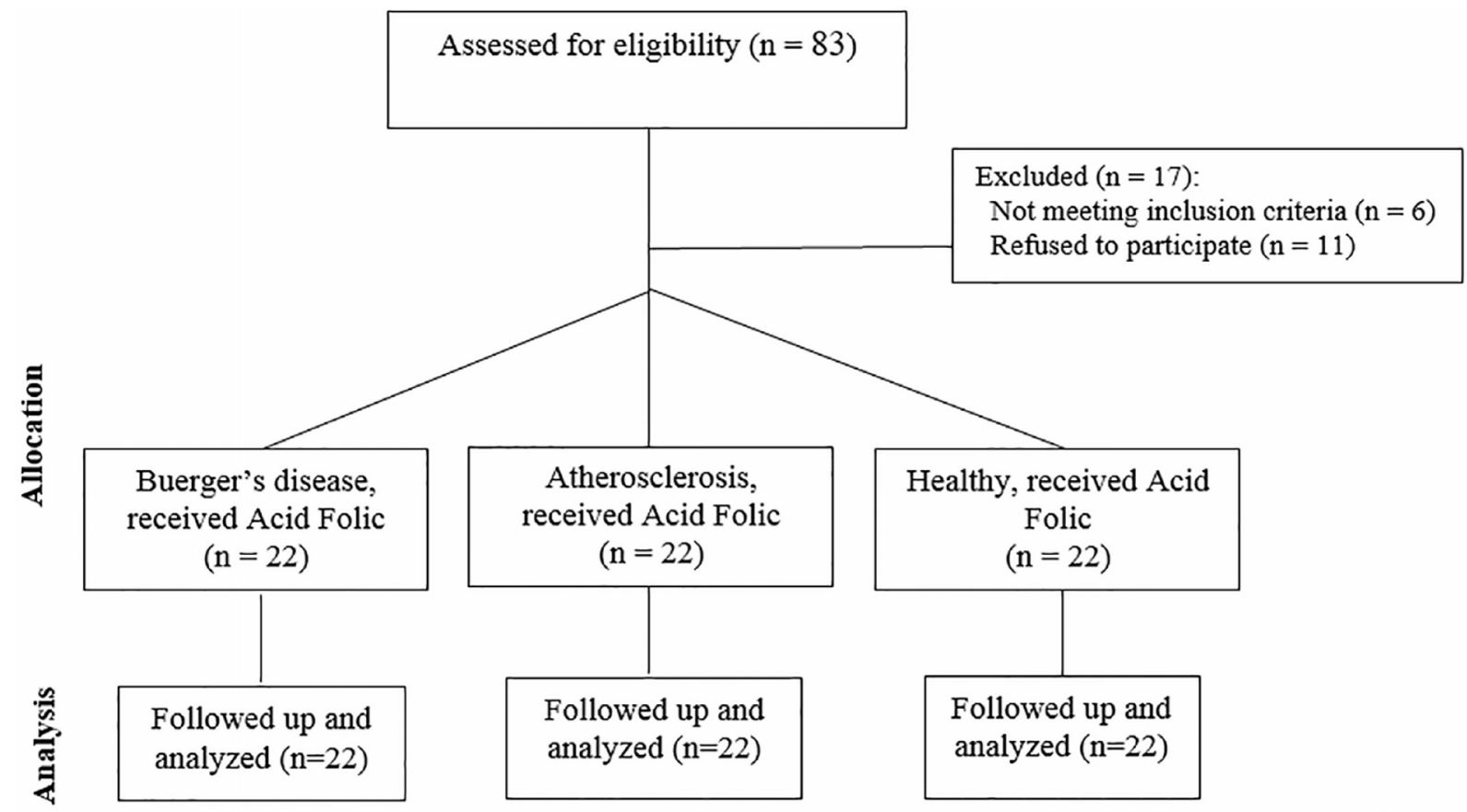

Figure1. CONSORT flow diagram of the study. 
Table 1. Underline co-morbidities in three groups.

\begin{tabular}{|l|l|l|l|}
\hline Co- morbidity, $\mathrm{n}(\%)$ & Healthy cases $(\mathrm{n}=22)$ & Atherosclerosis $(\mathrm{n}=22)$ & Buerger's disease $(\mathrm{n}=22)$ \\
\hline Hypertension & $2(9.1 \%)$ & $6(27.4 \%)$ & $2(9.1 \%)$ \\
\hline Diabetes & $1(4.5 \%)$ & $9(40.9 \%)$ & 0 \\
\hline Coronary artery disease & $1(4.5 \%)$ & $3(13.6 \%)$ & $1(4.5 \%)$ \\
\hline Cerebral artery disease & 0 & $1(4.5 \%)$ & 0 \\
\hline Renal failure & 0 & $1(4.5 \%)$ & 0 \\
\hline No co- morbidity & $18(81.9 \%)$ & $2(81.9 \%)$ & $19(86.4 \%)$ \\
\hline
\end{tabular}

Table 2. Initial and post treatment plasma homocysteine levels in 3 groups.

\begin{tabular}{|l|l|l|l|}
\hline Homocysteine level & $\begin{array}{l}\text { Buerger's disease }(\mathrm{n}= \\
22)\end{array}$ & $\begin{array}{l}\text { Atherosclerosis }(\mathrm{n}= \\
22)\end{array}$ & $\begin{array}{l}\text { Healthy group }(\mathrm{n}= \\
22)\end{array}$ \\
\hline Primary (micromoles per liter) & $21.8 \pm 8.5$ & $17.3 \pm 6.9$ & $13.8 \pm 3.1$ \\
\hline $\begin{array}{l}\text { After treatment (micromoles per } \\
\text { liter) }\end{array}$ & $14.6 \pm 4.5$ & $13.9 \pm 4.7$ & $10.7 \pm 3.9$ \\
\hline
\end{tabular}

\section{Discussion}

Multiple recent studies concluded that total hyperhomocycteinemia is a risk factor for atherosclerosis due to the disturbance of endothelial function and integrity $(1,2)$. Buerger's disease or Thromboangiitis obliterans (TAO) is a peripheral vascular disease with a questionable pathophysiology, natural course, and definitive treatment. Stammler (12) and Bergmark (13) have shown that the presence of high plasma levels of homocysteine in Buerger's disease patients. So, hyperhomocysteinemia may play a meaningful role in the pathophysiology of Buerger's disease. In our study, the plasma level of homocysteine was significantly higher in patients with Buerger's disease as compared to atherosclerosis and healthy cases before treatment. $(\mathrm{p}<0.001)$ The higher values of plasma homocysteine levels in Buerger's disease patients and simultaneously the higher prevalence of tobacco usage in this group ( $90.9 \%$ of cases) and also higher plasma levels of homocysteine in smoker cases of 3 groups was compared to the initial measurement in all patients, suggests that tobacco could be the principle cause of hyperhomocysteinemia in such patients, which also has been revealed in other studies $(4,6,12,14)$. The effect of folic acid therapy on elevated homocysteine levels in some medical conditions has been evaluated in a variety of studies. Van den Berg evaluated the effect of folic acid dosed at $5 \mathrm{mg}$ daily and vitamin B6 at 250mg daily therapy on homocysteine level in 72 patients with peripheral arterial occlusive disease and mild hyperhomocysteinemia. In $92 \%$ of the patients the post-load concentration was normalized and the fasting level in 91\% (15). Wilcken and colleagues were the first to demonstrate that a dose of $5 \mathrm{mg} / \mathrm{d}$ of folic acid can significantly reduce total free plasma homocysteine levels in patients with renal failure (16). Also, in a randomized, placebo controlled study, Bostom et al. demonstrated that a daily dose of $15 \mathrm{mg}$ of folic acid, B-6 (100 mg/day), and B-12 (1 mg/day) can lower total homocysteine plasma concentrations significantly, however the plasma level was normalized in only 5 out of 15 patients on regular hemodialysis treatment (17). Also, two meta-analysis studies have shown the important role of folic acid in reducing homocycteine levels which can lead to a decrease in vascular diseases $(11,18)$. Our study demonstrated a dramatic therapeutic response in the homocycteine level to a daily folic acid therapy $(5 \mathrm{mg} / \mathrm{d})$ in Buerger's disease and atherosclerotic patients, which can potentially affect the course of these diseases. One of our main limitations was the relatively small number of participants included in this study. Another limitation was that this clinical trial is not designed as a randomized study. Future randomized studies with a larger population could further validate our results.

\section{Conclusions}

In this study, we found that supplementation therapy with folic acid at $5 \mathrm{mg} /$ day significantly reduced the serum homocysteine level in Buerger's disease and atherosclerotic patients. Since Hyperhomocysteinemia is considered to be a risk factor in vascular diseases such as Buerger's disease, and according to the results of this study, the authors recommend more investigations into the details surrounding the homocysteine effects on the Buerger's disease progression, and even a discrete study to reevaluate the connection between smoking and hyperhomocysteinemia and then its correlation to Buerger's disease could yield additional information. This study measured the effects of folic acid only on the reduction of homocysteine level. Another discrete study is needed to analyze the effects of folic acid on natural course of the Buerger's disease. We recommend that folic acid should be considered as a routine agent in the Buerger's disease therapeutic regime. 


\section{Acknowledgments:}

We would like to thank the Vice Chancellor of Mashhad University of Medical Sciences for approving this project.

\section{Clinical trial registration:}

The trial was registered at the Thai Clinical Trials Registry (http://www.clinicaltrials.in.th) with the ID: TCTR20160601003.

\section{Funding:}

This study is not funded by any organization.

\section{Conflict of Interest:}

There is no conflict of interest to be declared.

\section{Authors' contributions:}

All authors contributed to this project and article equally. All authors read and approved the final manuscript.

\section{References:}

1) Ueland PM, Refsum H. Plasma homocysteine, a risk factor for vascular disease: plasma levels in health, disease, and drug therapy. J Lab Clin Med. 1989; 114(5): 473-501. doi: 5/1/15088. PMID: 2681479.

2) Den Heijer M, Rosendaal FR, Blom HJ, Gerrits WB, Bos GM. Hyperhomocysteinemia and venous thrombosis: a meta-analysis. Thromb Haemost. 1998; 80(6): 874-7. PMID: 9869152.

3) Božič-Mijovski M. Hyperhomocysteinemia and thrombophilia. Clin Chem Lab Med. 2010; 48(1): 89-95. doi: 10.1515/CCLM.2010.365. doi: 10.1515/CCLM.2010.365. PMID: 21105837.

4) Di Micco P, Di Fiore R, Di Micco G, Cardillo G, Bellia C, Quaranta S, et al. Buerger's Disease and Hyperhomocysteinemia: Is there a Relationship? J Atheroscler Thromb. 2008; 1: 6-8. doi: 1876-5068/08.

5) Fiessinger JN. Juvenile arteritis revisited. Buerger's disease-Takayasu's disease. Pathophysiol Haemost Thromb. 2002; 32(5-6): 295-8. doi:10.1159/000073584. PMID: 13679660.

6) Ravari H, Zafarghandi MR, Alvandfar D, Saadat S. Serum homocysteine in deep venous thrombosis, peripheral atherosclerosis and healthy Iranians: a case-control study. Pak J Biol Sci. 2009; 12(14): 1019-24. doi: 10.3923/pjbs.2009.1019.1024. PMID: 19947180.

7) Sunder-Plassmann G, Födinger M, Buchmayer H, Papagiannopoulos M, Wojcik J, Kletzmayr J, et al. Effect of High Dose Folic Acid Therapy on Hyperhomocysteinemia in Hemodialysis Patients Results of the Vienna Multicenter Study. J Am Soc Nephrol. 2000; 11(6): 1106-16. doi: 1046-6673/1106-1106. PMID: 10820175.

8) Koyama K, Usami T, Takeuchi O, Morozumi K, Kimura G. Efficacy of methylcobalamin on lowering total homocysteine plasma concentrations in haemodialysis patients receiving high - dose folic acid supplementation. Nephrol Dial Transplant. 2002; 17(5): 916-22. doi: 10.1093/ndt/17.5.916. PMID: 11981084.

9) de Jong SC, Stehouwer CD, van den Berg M, Kostense PJ, Alders D, Jakobs C, et al. Determinants of fasting and post-methionine homocysteine levels in families predisposed to hyperhomocysteinemia and premature vascular disease. Arterioscler Thromb Vasc Biol. 1999; 19(5): 1316-24. doi: 10.1161/01.ATV.19.5.1316. PMID: 10323785.

10) Nygård O, Nordrehaug JE, Refsum H, Ueland PM, Farstad M, Vollset SE. Plasma homocysteine levels and mortality in patients with coronary artery disease. N Engl J Med. 1997; 337(4): 230-6. doi: 10.1056/NEJM199707243370403. PMID: 9227928.

11) Boushey CJ, Beresford SA, Omenn GS, Motulsky AG. A quantitative assessment of plasma homocysteine as a risk factor for vascular disease: probable benefits of increasing folic acid intakes. JAMA. 1995; 274(13): 1049-57. doi: 10.1001/jama.1995.03530130055028. PMID: 7563456.

12) Stammler F, Diehm C, Hsu E, Stockinger K, Amendt K. The prevalence of hyperhomocysteinemia in thromboangiitis obliterans. Does homocysteine play a role pathogenetically? Dtsch Med Wochenschr. 1996; 121(46): 1417-23. doi: 10.1055/s-2008-1043162. PMID: 8974873.

13) Bergmark C, Mansoor MA, Swedenborg J, de Faire U, Svardal AM, Ueland PM. Hyperhomocysteinemia in patients operated for lower extremity ischaemia below the age of 50-effect of smoking and extent of disease. European Journal of Vascular Surgery. 1993; 7(4): 391-6. doi: 10.1016/S0950-821X(05)80255-5. PMID: 8359294. 
14) Van den Berg M, Boers GH, Franken DG, Blom HJ, Van Kamp GJ, Jakobs C, et al. Hyperhomocysteinaemia and endothelial dysfunction in young patients with peripheral arterial occlusive disease. Eur J Clin Invest. 1995; 25(3): 176-81. doi: 10.1111/j.1365-2362.1995.tb01545.x. PMID: 7781664.

15) van den Berg M, Franken DG, Boers GH, Blom HJ, Jakobs C, Stehouwer CD, et al. Combined vitamin B< $\mathrm{sub}>6</$ sub $>$ plus folic acid therapy in young patients with arteriosclerosis and hyperhomocysteinemia. J Vasc Surg. 1994; 20(6): 933-40. doi: 10.1016/0741-5214(94)90230-5. PMID: 7990188.

16) Wilcken DE, Dudman NP, Tyrrell PA, Robertson MR. Folic acid lowers elevated plasma homocysteine in chronic renal insufficiency: possible implications for prevention of vascular disease. Metabolism. 1988; 37(7): 697-701. PMID: 3386535.

17) Bostom AG, Shemin D, Lapane KL, Hume AL, Yoburn D, Nadeau MR, et al. High dose B-vitamin treatment of hyperhomocysteinemia in dialysis patients. Kidney Int. 1996; 49(1): 147-52. doi: 10.1016/0026-0495(88)90093-5. PMID: 8770960.

18) Collaboration HLT. Lowering blood homocysteine with folic acid based supplements: meta-analysis of randomised trials. BMJ. 1998; 316(7135): 894-8. doi: 10.1136/bmj.316.7135.894. PMID: 9569395, PMCID: PMC28491. 